\title{
Images and Illustrations to Build the Meaning in Arabic Dictionaries
}

\author{
Hussein Mohammad Bataineh $^{1 *}$, Abdallah Hussein El-Omari ${ }^{1}$ \\ ${ }^{1}$ Al-Balqa' Applied University, Jordan \\ *Corresponding author: Abdallah Hussein El-Omari: d.abdullah.amri@gmail.com
}

Citation: Bataineh H.M., El-Omari A.H. (2018) Images and Illustrations to Build the Meaning in Arabic Dictonaries. Open Science Journal $3(2)$

Received: $24^{\text {th }}$ February 2018

Accepted: $28^{\text {th }}$ March 2018

Published: $20^{\text {th }}$ April 2018

Copyright:@ 2018 This is an open access article under the terms of the Creative Commons

Attribution License, which permits unrestricted use, distribution, and reproduction in any medium, provided the original author and source are credited.

Funding: The author(s) received no specific funding for this work

Competing Interests: The author have declared that no competing interests exists.

\begin{abstract}
:
Current requirements to get dictionaries cope with the consequences of both the scientific and digital revolutions emphasize the importance of the methods to convey the accurate, exact and clear meaning which should not bear any error or the possibility of error to the recipient. Therefore, images and illustrations are among the means which are imposed on the science of lexicography making. That is why this research sheds light on this technique considering its nature, importance, peculiarities, functions, defects, and technicalities. The research also investigated the reality of images and illustrations in contemporary Arabic dictionaries and the difference between them and ancient ones.
\end{abstract}

Keywords: Dictionaries, Entries, Images, Illustrations

\section{Introduction}

Scholars of psychology and semantics divide meanings and concepts into concrete tangible and mental abstract meanings. Each one of these meanings has its own method to approach. The concrete tangible is the one that is realized by the senses, whilst the mental abstract meaning is isolated from material and all its relatives. The tangible or external world is a group of things which are recognized by the senses. The mental or internal world is something that is related to mentality. Tangibles are divided into specific which are recognized by a specific sense of man such as tastings or hearings, and common senses which are widely recognized (Qasemi, 2008, p. 326). No doubt that approaching the 
concrete tangible is easier than the abstract items as they are very much closer to mentalities than the abstract. However, this does not mean the impossibility of approaching lexical items which convey abstract meanings. Nevertheless, expressing abstract meanings would be easier, but generally speaking, they remain more difficult. More importantly, age categories approaching the lexical item should be considered here. These are usually divided into three main categories: children, youths, and adults. To be more specific, these also can be subdivided. Children for example, can be divided into those below six years of age, and those over. The same can be said about the other age categories.

Another perspective can be considered in this regard, which is merely one of the criteria that are used to approach the meaning and methods of constructing and formulating it. This criterion is established upon a cognitive basis, i.e., not all age categories are scientifically and culturally equal. With children, for example, there are differences and changes between a child and another. Some children surpass their peers intelligently, scientifically, and educationally. The same can be said about the youth and adult categories; as there are the illiterate, literate, and educated on the one hand, and the scientists, the creative, the inventors, and the distinguished on the other hand. Therefore, all these issues have to be considered when meanings are constructed in dictionaries.

This implies and means that each age category requires its own dictionary, and children, and pupils of elementary schools should at least have special dictionaries. It is worth noticing here that specialized scientific dictionaries which are concerned about scientific and technical terms of medicine, biology, engineering, etc.. highly require supporting their scientific concepts with pictures and illustrations.

\section{Psychological dimension of meaning}

There are several factors that make the meaning different, among of which are the recipients' culture, and their psychological and social status. Authorized techniques of constructing and actualizing it completely and clearly to the recipient affect the structuring of the concept. The meaning is a fixed substance around which there are semantic expansions that would extend or shrink according to the cognitive reservoir of the recipient.

Regardless of the disputes about the definition and identification of a word, it has an almost continuous meaning. Some people see that a word is meaningless out of context. Its meaning, in fact, is identified in the context it appears in. Among the most important methods of structuring the meaning in dictionaries in general, are images which receive as well as convey the meaning.

\section{Research Methodology}

This research employs the analytical descriptive semiotic approach to conduct a factual realistic survey. It analyzes the readability of Arabic dictionaries in recent and ancient times from a modern semiotic perspective with all its various theories (Omar, 1992, p, 54). Using images and illustrations in dictionaries is 
explained and investigated to reflect the importance of these means for dictionaries and those who use them.

\section{Illustrations}

Structure of the meaning in the dictionary differs from its structure in context or use outside the dictionary. Dictionary is a legible envisioned written material. Therefore, it is easy to control lexical entries and units and scientific terms as well as structuring their concepts and building their meanings by many methods and techniques, which are mainly related to language i.e. linguistics. Some of them however, are nonlinguistic such as illustrations and helping pictures, figures and drawing examples. Illustrations in lexical fields mean: every nonlinguistic sign which explains a linguistic meaning reference. That is why it includes the symbols, forms, icons, features and drawings. Therefore, words and drawings are two cultural signs that differ in form but look the same in content. They have remained (words and drawings) two integral poles, where one of them recalls the other in the human communication semiotic and linguistic systems through successive ages (Jilale, 1999, p. 226).

Since the smallest meaningful linguistic unit is the morpheme, the smallest meaningful visual unit will be the dot or period. The linguistic feature (linguistic symbol) meets the visual one (illustration) within the poles of the semantic triangle. However, the denotation in the first is the written or spoken word, and the denoted is the mentally envisioned. On the other hand, the denotation in the second is the form seen by the eye such as a picture of an animal on paper, and the denoted is the idea or concept that the picture or illustration symbolizes (ibid, 226). The difference between the linguistic and nonlinguistic denotation is that the first one is irregular and has no relation with its meaning, whereas the relation of the second one with its meaning is not irregular.

Fleming (in Jilale, 1999, p. 227) generally introduces the illustration as the formations of the written line, dot and area or any form of these three elements resembling events or actions - persons, places or things - whether being seen or conceived (Zafanky, 2007, p. 293). Using pictures and drawings in dictionaries is a sort of encyclopedic information since such means show interest in the area of investigation rather than the linguistic symbol itself. Therefore, they are naturally encyclopedic because the drawing or the picture compared to the term is similar to the concerned synonym or definition of the general word (ibid, p. 293). Image entries have been firstly used in 1897 when John Amos Koveos (in Zafanki, 2007, p. 293) issued his bilingual book entitled 'The World in Images' (ibid), whereas in the dictionary, it was firstly used by the French Forepiere in 1868 (ibid, p.293). Arabs have had their own tries in this field which can not be ignored. It is worth mentioning that these efforts have been more dedicated to school dictionaries such as the 'Manhal Allughah' (Source of Language) dictionary 1997, then 'Al Qamous Al Musawar' (Dictionary in Images) 1999, and 'Al Mu'jam Al Mutamaiyez Al Majjani Al Musawar' (The Distinguished Imaged Free Dictionary) 2000, and others. 


\section{Types of image features}

Kokella (in Jilale, 1999) proposes that images are categorized into:

1. Icon, which is different from linguistic features, that there is an assimilation relationship between it and the meaning, i.e., the picture of a car is similar to the car in reality.

2. Non-icon, which is semi-irregular and close to linguistic features, it does not accept dual interruption, though. This means that the meaning is not fully like the real reference although it is not verbal. It is made up of dots, lines, and different symbols such as the punctuation marks, numbers, arithmetic symbols and the like.

3. Vague image features, where forms and figures intersect to a degree that it becomes difficult to recognize, such as square art paintings and abstract art. This type rarely contributes in defining entries lexical units because of its high vagueness and need of explanation and interpretation (Jelali, p. 227).

\section{Meaning features and functions of images and illustrations}

Zafanky (2007, p. 293) believes that images and illustrations have meaning features and functions which the others do not possess, as they are:

1. More descriptive of the meaning than expressions and linguistic signals.

2. Sometimes more space saving than linguistic definition.

3. More capable of recognizing different forms of the same type than the expression itself.

4. Has a psychological and educational appearance.

5. Enhance the intended meaning in the recipient's mind as it truly links the signal and the meaning,

6. Remedies a defect in the meaning and increases clarity of the word meaning.

However, this does not mean that the technicality of illustrations is ultimately typical. In Arabic dictionaries they suffer from contradictions and defects which eliminate their importance and functional role. This technicality also becomes unable to hold the burdens of the meaning alone. This means that it remains an assisting and complementary method in constructing the meaning, i.e. a subsidiary one. Among the defects of illustrations and pictures are the following (ibid, p. 294):

1. They are set as entries of sciences and arts, and they care about items rather than articulations.

2. Their applied areas are limited such as material items which have clear and distinctive form.

3. There are many material items which are uneasily displayed in clear forms such as the acids. 
4. Being sometimes unable to separate and recognize similar concepts, as there are many pictures whose nature cannot be realized unless they are explained (ibid, p. 295).

\section{Types of image examples}

Based on categories of sight features, these features appear in the dictionary in different forms:

1. Drawing: it means representing or personifying things of an area with writing or crafting methods (Jilale, 1999, p. 230). Al-Wasit dictionary stated that drawing is representing something or person with a pen or the like (1973). Therefore, drawing an item on paper would not be typically matching the real physiology of the meaning. However, this does not mean that the relationship between the drawing and its meaning is irregular. It is rather real, since typicality is semi-complete and approximate.

2. Image: As defined in Al-Wasit dictionary, it is the form and casted statue. There are two types of images: traditional image and photo. The latter is better than the traditional because it is accurate and matches reality especially colored ones.

3. Scheme: It is a form or simple graph focusing on the core of the meaning, and formulates its prominent features. This definition states that the scheme is a complex form which takes the basics of the body or the item without highlighting the minute details (Jurr, 1987, p. yd). This type of sight features is approximate and semi-typical.

\section{Functions of image examples in the dictionary}

Different images and illustrations have various important functions in building the meaning and presenting it to the recipient. The most prominent functions may be limited in the following:

1. Psychological: An image answers the human being's psychological need when he / she resort to reality rather than language whenever this has been possible. It has been proven that man thinks with the mental image. That is why when he / she read, they change ideas into a mental image (Jilale, 1999, 232). An image satisfies man's curiosity, as hearing would never be as beholding. Man naturally prefers seeing and approaching items. Dolacroa in 1863 (ibid, p. 232), states that an image has a complementary function to words, and more importantly we can imagine it without words. Many people prefer images to words (ibid, p. 232).

2. Defining: It is the most important function a dictionary specialist endeavors to achieve through confirming the use of images and illustrations which carry an educational and instructional objective, especially when introducing an entry that is difficult to explain by words (ibid, 232). This view is truly reasonable and correct, as there 
are many situations at which we look helpless about explaining the meaning of some words or terms. Therefore, we seek the help of images, hand signals, or wave drawings in the air. Some people believe that language sometimes looks unable to approach information about, for example making a knot, using a method, or studying blood circulation says Atchison (2009, p. 34). The English writer, Heller Yieblock (Auden, 1962-1972, p. 34) said: If you can describe how to make a knot clearly without having to use illustration, you are very skillful in using the English language. Atchison (2009, p. 35) concludes: An image is worth a thousand words. Language is short to express place information and feelings (ibid, p. 36). An image is capable of showing minute details and distinguish similar items, and honestly, accurately, and objectively transferring things considering their dimensions, measurements, forms, colors having them in order and coordination which make them understandable and away from vagueness (Jelali, 1999, p. 232).

3. Aesthetic: Compared to the previous functions, it comes third in importance. It is among the complementary and supplementary functions. It is an attractive one which is worth seeing, and kindles interest which makes a dictionary attractive for consulting and commercially promoted (Lajme, 1993). These images are not decorative, though, specially the colored ones. They are not meant to attract the eye either, but to support definition, and colors are employed to recognize differences among similar items of flowers, birds fish and others (Hlaiyel, 2010).

\section{Image techniques and specifications in the dictionary:}

Images and illustrations have no value unless they achieve necessary specifications and conditions. Importance does not lie in disordered representation of the meaning by an image. What is important here is the quality of the specific image and how it meets the required specifications and conditions, so that it can be directed and displayed by the nearest probable image of the real meaning. However, Arabic dictionaries with images have suffered from defects preventing them from performing the tasks which they were expected to do, misusing images, or unneeding them in some other cases (Hlaiyel, 2010). Hlaiyel (2010) believes that images or drawings of Al M'ujam Al Wasit dictionary completely failed since readers did not recognize or identify a tree, flower, or bird. He also believes that dictionary making needs an experience and knowledge of the objectives of drawing and imaging to explain things, actions, relationships, forms, qualities, movements, appliances, animals, fish, vegetables, historical and civilization items, and species such as kinds of snakes, birds, or flowers that belong to the same semantic field (ibid). The most prominent specifications and conditions are:

1. Accuracy: It is achieved by having no difference between the item and its image regarding form, color, and dimension. An error in image definition is more serious than a verbal one (Jilale, 1999, p. 240). 
2. Clarity: It is verified by highlighting the features of the imaged item so that the reader can realize and understand the content of the image (ibid, p. 240). The image can be supported with captions, symbols, summaries, arrows, and different colors. Here, each part might be given a different color or number or any other signal to supplement the function of the image.

3. Brevity: The imaged example should be exclusively used to highlight the main elements of the defined item, so that the image does not take big space which might distract the attention of the recipient (ibid, p. 240). Therefore, a standard size is to be adopted for all images in the dictionary, so they take nearly the exact area.

4. Type: Images which mean the same thing are many. For example, the word 'armchair' can be explained by many images. However, these images are not always of the same quality. Images should be carefully selected to explain the meaning. Dictionaries should refer readers to the whereabouts of the images if they are not near definitions so that they would not be drawn again. The image of the eye, for example, should be provided with all its parts (lens, optical nerve, pupil etc.), so the dictionary do not have to provide the image of the eye again when the entry word is (pupil or optical nerve) (ibid, p. 241).

5. Size: Image size is relatively not fixed because quality of the image decides its size. There are single and simple images, but there are complex and complicated ones. There are also serial images which explain, for instance stages of manufacturing something, phases of disease progression, or stages of fetal growth. These will inevitably take greater area than other images. Image size is also subject to quality and objective of the dictionary. Among the techniques to be considered about selecting the size of the image:

a. The size of the image that has a single meaning should not be less than $[1 / 1.5 \mathrm{~cm}]$, and not more than $[6 / 10 \mathrm{~cm}]$ if it were complex or in series. Exceeding these limits, a whole page shall be specifically given, or inserted as posters inside dictionary text, or outside it as appendixes.

b. The image can be made smaller or enlarged considering the length and width according to some mathematical calculations. If the original size of an image for example is 50/20 and the required length in the dictionary is $5 \mathrm{~cm}$, the width will be $2 \mathrm{~cm}$ (ibid, p. 242).

6. Number: This is the number of approved images in one dictionary, which is a relative criterion too. There is no rule for the number of images in a dictionary compared to the number of entries since the images are merely helping methods but not a linguistic verbal definition. This means that the number of images and illustrations is not subject to any condition or agreement. The number of images and illustrations is necessary as long as the linguistic analysis is unable to give a clear and expressive definition (ibid, p. 242).

Therefore, when the editor of the third international Webster Dictionary wanted to add about ten thousand new meaning entries which have not existed in the previous edition, they decided to reduce 
the number of colored posters and drawings to a ratio of one third or a quarter (ibid, p. 242).

7. Color: It is an important issue in this regard as it motivates following up details of the image, clarifies particles of the meaning, and attracts it to its natural reality. The value of color varies from one dictionary to another and from a definition to another. Some definitions need color more than others, especially those definitions in which color becomes the sole distinct peculiarity as for example with some chemicals, blood groups, types of flowers, birds, lights and colors in specific (ibid, 242). This difference emerges from the reality that color has a direct contact with the pedagogic and educational aspects of the dictionary, which makes the colored images clearer than the black and white ones as well as better in conveying the true meaning of words. Colors cannot absolutely be excluded in clarifying the meaning behind issues related to color grades or any color explanation (ibid, p. 242).

\section{Images and Illustrations in Contemporary Arabic Dictionaries:}

There are differences between old and modern images and illustrations in Arabic dictionaries. Old dictionaries had simple and few drawings, whilst modern and contemporary dictionaries abundantly and extensively use images. Reviewing contemporary famous dictionaries such as Al-Munjid, Al-Waseet, Al-Qamous AlJadeed for Students, Al-M'ujam Al-Arabi Al-Hadeeth, and Al-Moheet, it would be found that they had shortcomings and defects regarding the images and illustrations' adopted technique. These shortcomings and defects could be summarized in the following:

1. Not following systematic scientific approach in using the images and illustrations regarding quantity or quality and importance, as they are helping methods to define lexical entries difficult to explain.

2. Some drawings are sometimes approximately sketched and hand drawn which distorts the imaged item.

3. The dictionary approved many wrong and inaccurate drawings expressing other unintended items as definitions. Therefore, many mergence and repetitions occurred.

4. The dictionary does not sometimes depend on complex, serial and colored images.

5. Neglecting many images which are worth keeping, and retain some which are not needed.

6. Meager ratio of images and too few to match the number of vocabulary entries in the dictionary.

7. Misplacement of images, entries, inaccuracy, and wrong ratio of images to intended entries.

8. Small size of images, since some are not more than 1 square centimeter (ibid, p. 246).

These defects and shortcomings might be found in Al-Raed dictionary by Jubran Masoud (1978) which contains only 32 illustrations. They are common 
and popular among modern and contemporary Arab dictionaries which can be easily avoided by individuals and establishment. They can realize their minute causes and work seriously to produce model and ideal images which properly and accurately reflect the meaning. In fact, they are not excused for not doing so, as computers and electronic devices are equipped with very high technologies and programs which can process images and illustrations in various methods. The web nowadays has facilitated access to knowledge and information to carry out application and processes to produce high level images and illustrations. Developed printers and printing methods, as well as the high quality of paper, packaging and classification have added to the betterment of images and illustrations production.

Digital dictionaries might have largely surpassed paper dictionaries in this regard. It is very probable to insert motion images and films displaying the meaning accurately.

Therefore, image and illustration are among the most important elements and constructions which clarify scientific, artistic and technical meanings since it is said that one picture is worth a thousand words.

\section{References:}

Al-Waseet Dictionary, the Academy of Arabic Language, Cairo, Dar Al-Ma'aaref Press, Egypt, $2^{\text {nd }}$ Edition, 1973.

Atchison,J,(2009), Speech seeds, language origin and development, Translated by Wafeeq Faiq Kreishat, Publications of Syrian General Book Organization, Ministry of Culture, Damascus.

Auden, W. H. and L. Kronenberger $(1962$ - 1970), The Faber Book of Aphorisms, Londontur: Faber Paperbacks.

Hlaiyel, M. M. H. (2008), Towards a Contemporary Arabic Dictionary, Arab Organization for Education, Culture and Sciences, April.

Jilale, Hallam. (1999), Techniques of introducing modern Arab dictionaries, Publications of Arab Writers Union.

Jurr, K. (1987), Modern Arabic Dictionary (La Ross), La Ross Library, Paris.

Lajmi, Adeeb and others (1993), Al-Muheet Arabic Dictionary, Dar Al-Muheet, Paris.

Omar, Ahmad Mukhtar, (1992), Theoretical and Conceptual Theory, Semantics, Book World, $3^{\text {rd }}$ Edition, Egypt.

Qasemi, Ali (2008), Terminology "Theoretical Bases and Practical Applications", Lebanon Library Publishers, $1^{\text {st }}$ Edition, Beirut, Lebanon.

Raed, Jubran Masud (1978), Dar Al-Ilm Lilmalayeen, $3^{\text {rd }}$ Edition, Beirut.

Zafanki, Safiah, (2007), Lexical developments and modern general Arab linguistic dictionaries, Publications of Ministry of Culture, Syrian General Book Organization, Damascus. 